\title{
Aspectos da distribuição e conservação de Paspalum L. (Poaceae) em Pernambuco ${ }^{1}$
}

\author{
Jefferson Rodrigues Maciel ${ }^{2,5}$, Regina Célia de Oliveira ${ }^{3}$ e Marccus Alves ${ }^{4}$
}

Recebido: 09.12.2009; aceito: 28.12.2010

\begin{abstract}
Aspects of distribution and conservation of Paspalum L. (Poaceae: Panicoideae in Pernambuco). Given the high richness and importance of Paspalum for the flora of Pernambuco, this work aims to describe the distribution of species of this genus in the state and contribute to the identification and understanding of the environmental factors that influence the distribution of the species and its conservation. With the DIVA-GIS software, maps were developed of the following parameters: species distribution, overlay of species richness by phytogeographic subdivision and a model to predict the areas with favorable climatic conditions for the establishment of the examined species. The species documented with three or fewer records and/or restricted to one location were classified as rare. Paspalum occurs widely in Pernambuco, but the distribution of most species is limited by increased seasonality in the east-to-west direction. In the region that includes the restinga, mata úmida, mata seca and agreste subzones all analyzed species are found. The subzone of the mata úmida (moist forest) had the largest number of species, followed by restinga (coastal) and the subzone agreste (transition zone between moist and semi-arid regions). The modeling revealed two regions with optimal environmental conditions for the species. These areas are susceptible to human action. Twelve species were classified as rare, occurring mainly in the more humid regions where an area was identified with optimal conditions for the establishment of populations of many species.
\end{abstract}

Key words: DIVA-GIS, Geographic Information System, grasses, modeled distribution

RESUMO - (Aspectos da distribuição e conservação de Paspalum L. (Poaceae) em Pernambuco). Diante da elevada riqueza e importância de Paspalum para a flora de Pernambuco, este trabalho teve como objetivo descrever a distribuição do gênero no Estado e identificar fatores ambientais que potencialmente influenciam na distribuição das espécies, além de abordar a atual situação da conservação das espécies do gênero no Estado. Com o software DIVA-GIS foram elaborados mapas de distribuição das espécies, grids de riqueza por zona e subzona fitogeográfica e uma modelagem previsiva das áreas com melhores condições climáticas para o estabelecimento das espécies analisadas. As espécies com até três registros e/ou restritas a uma localidade foram classificadas como raras. Paspalum ocorre amplamente em Pernambuco, mas a distribuição da maioria das espécies é limitada pelo aumento da sazonalidade no sentido leste-oeste. No trecho que compreende as subzonas da restinga, da mata úmida, da mata seca e do agreste são encontradas todas as espécies analisadas. A subzona da mata úmida apresentou o maior número de espécies, seguida pela subzonas da restinga e do agreste. A modelagem ambiental revelou duas regiões com condições ótimas para as espécies. Estas se encontram em áreas susceptíveis à ação antrópica. Foram registradas doze espécies raras, que ocorrem principalmente nas regiões mais úmidas onde também foi identificada uma área com condições ótimas para o estabelecimento de populações.

Palavras-chave: distribuição modelada, DIVA-GIS, gramíneas, Sistema de Informação Geográfica

\section{Introdução}

O Estado de Pernambuco possui uma área de $98.079 \mathrm{~km}^{2}$, com maior extensão no sentido longitudinal e segundo Andrade-Lima (1960), não apresenta tipos particulares de vegetação exclusivas de seu território, mas apenas continuações de grandes zonas fitogeográficas do Brasil. Esse autor divide
Pernambuco em quatro zonas fitogeográficas: zonas do litoral, da mata, da caatinga e das savanas, as quais foram delimitadas de acordo com peculiaridades florísticas e abióticas.

Paspalum L. está representado por 32 espécies no Estado, das cerca de 330 que possui (Maciel et al. 2009a, b), e é caracterizado pelas espiguetas planoconvexas e adaxiais, distribuídas unilateralmente na

1. Parte da dissertação de Mestrado do primeiro autor, Programa de Pós-Graduação em Biologia Vegetal-PPGBV da Universidade Federal de Pernambuco

2. Prefeitura da Cidade do Recife, Jardim Botânico do Recife, Km 7,5 da BR 232 s/n, 50000-230 Recife, PE, Brasil

3. Universidade de Brasília, Herbário, Caixa-Postal 4457, 70919-970 Brasília, DF, Brasil

4. Universidade Federal de Pernambuco, Laboratório de Morfo-Taxonomia Vegetal, Av. Moraes Rego s/n, $50670-930$ Recife, PE, Brasil

5. Autor para correspondência: jeff.r.maciel@gmail.com 
ráquis, além da ausência de gluma inferior na maioria das espécies (Clayton \& Renvoize 1986). Ocorre principalmente nas regiões tropicais e subtropicais das Américas, sendo seu centro de diversidade o cerrado brasileiro (Rua 2006). A grande diversidade de Paspalum é refletida no seu amplo espectro de preferências ecológicas. Suas espécies ocorrem desde o nível do mar até altitudes superiores a $4.500 \mathrm{msm}$ (Morrone et al. 1995, 2000), em bordas de matas, áreas alteradas e savanas. O gênero possui espécies aquáticas e adaptadas à aridez ou salinidade relativamente alta (Barreto 1957, Clayton \& Renvoize 1986).

O conhecimento detalhado da distribuição de espécies tem sido empregado no sentido de otimizar os esforços de coleta de germoplasma e para estabelecer ações prioritárias para o manejo e conservação de determinadas espécies ou táxons acima do nível específico, bem como para identificar os padrões de biodiversidade em determinadas regiões (Arrieta \& Ortiz 2009, Chiron 2009, Jarvis et al. 2003, Hijmans \& Spooner 2001). Essa tendência reforça a importância de estudos regionalizados da distribuição de táxons, sobretudo para desenvolver e detalhar o conhecimento da riqueza de espécies em um país reconhecido por sua megadiversidade biológica.

Diante do exposto, o objetivo deste trabalho é descrever a distribuição de Paspalum em Pernambuco e identificar fatores ambientais que influenciam na distribuição das espécies, além de abordar a atual situação da conservação das espécies do gênero no Estado.

\section{Material e métodos}

Para este trabalho foram realizadas coletas em diversas regiões fitogeográficas de Pernambuco entre janeiro de 2006 e agosto de 2008. Os dados também foram coletados no banco de dados do Catalogue of New World Grasses-CNWG (Soreng et al. 2000), nas revisões do gênero (Chase 1929, A. Chase, dados não publicados, Denham 2005, Morrone et al. 1996, R. C. Oliveira, dados não publicados, Zuloaga \& Morrone 2005) e nas análises das coleções depositadas nos herbários ASE, BOTU, CPATSA, CEN, HST, HUEFS, IBGE, ICN, IPA, JPB, MOSS, PACA, PEUFR, RB, SP, SPF, UB e UFP (siglas conforme Thiers 2010, exceto CPATSA, Herbário da Embrapa Semi-Árido, e HST, Herbário Sérgio Tavares da Universidade Federal Rural de Pernambuco).

Foram analisadas 31 espécies neste estudo, tendo sido excluído Paspalum notatum Fluggé por se tratar de espécie introduzida que só é encontrada em canteiros e jardins (Maciel et al. 2009a, b). Os registros foram georreferenciados no campo ou com o auxílio de imagens de satélite e da ferramenta de correção de georreferenciamento do DIVA-GIS. Esses dados foram visualizados com o software DIVA-GIS (Hijmans et al. 2001). Como mapas base foram utilizados os recursos disponíveis pelo catálogo de mapas base da ESRI (Enviromental Systems Research Institute), do banco de dados DIVA-GIS (disponível em http://www.diva-gis.org/Data.htm) e de Áreas Prioritárias para Conservação no Brasil do Ministério do Meio Ambiente-MMA (MMA 2007). O mapa base fitogeográfico de Pernambuco foi digitalizado a partir do mapa publicado por AndradeLima (1960).

Os dados georreferenciados foram plotados no mapa fitogeográfico de Pernambuco para análise da distribuição das espécies. $\mathrm{O}$ número de espécies por subzona fitogeográfica foi sumarizado com o auxílio do DIVA-GIS. Foram criados grids de riqueza de espécies com células de $1^{\circ}$ por $1^{\circ}$, seguindo parcialmente o processo descrito por Hijmans \& Spooner (2001). Por fim, a distribuição potencial das 31 espécies de Paspalum analisadas neste estudo foi modelada com o algoritmo Bioclim, tendo por base as 19 variáveis bioclimáticas do banco de dados worldclim numa resolução de cinco minutos por pixel, utilizando a versão 1.3 (disponível em http://www.diva-gis.org/climate.htm). Essas variáveis advêm de valores mensais de temperatura e precipitação, representado médias, sazonalidade e condições extremas de temperatura e precipitação ao longo do ano, as quais são amplamente utilizadas em estudos de modelagem de nicho ecológico (Hijmans et al. 2005). Os dados da distribuição conhecida utilizada na modelagem foram obtidos das coletas realizadas no Estado, do levantamento no CNWG (Soreng et al. 2000) de levantamentos de herbários, e na bibliografia disponível para o gênero.

As espécies com até três registros ou com distribuição restrita a uma ou duas localidades foram classificadas como raras. Estas espécies tiveram suas distribuições analisadas seguindo os mesmos métodos descritos acima. Para elas também foram modeladas as prováveis distribuições com base em variáveis climáticas.

Para análise da distribuição das espécies no contexto fitogeográfico pernambucano foi utilizada a classificação fitogeográfica do Estado proposta por Andrade-Lima (1960). Nessa classificação o autor propõe quatro zonas fitogeográficas (figura 
1a), as quais são divididas em subzonas tendo como parâmetros profundidade e textura do solo, altitude, distribuição das chuvas, riqueza florística, fisionomia e perenidade das folhas. Sendo assim, a zona do litoral é dividida em vegetação marinha, de praia e restinga. A zona da mata é dividida em mata úmida, seca e serrana. A zona da caatinga é dividida em agreste e sertão. A subzona do sertão é dividida em regiões denominadas sertão central, sertão dos chapadões cretáceos, sertão do São Francisco e Sertão do Jatinã. E a zona das savanas é dividida em tabuleiros e sertão do Araripe (figura 1b). Por questão de escala a Figura $1 \mathrm{~b}$ representa a zona do litoral sem mostrar suas subdivisões.

\section{Resultados e Discussão}

As espécies do gênero Paspalum se encontram amplamente distribuídas em Pernambuco (figuras 3,
4), ocorrendo em quase todas as zonas e subzonas fitogeográficas do Estado, sendo a exceção a subzona dos tabuleiros, um padrão que se repete em diversos grupos já estudados em Pernambuco (Abreu et al. 2008, Costa-eSilva \& Maciel 2007, Melo \& Sales 2004, Silva \& Sales 2007, Souza \& Sales 2004). Pela figura 2a percebe-se que as subzonas da restinga, da mata úmida, mata seca e agreste abrigam todas as 31 espécies de Paspalum analisadas neste estudo (tabela 1).

Na figura 2a também se observa que a subzona da mata úmida é a que apresenta o maior número de espécies dentre as subzonas fitogeográficas do Estado. $\mathrm{Na}$ mata úmida são encontradas 21 espécies, uma concentração elevada que enfatiza a importância desta subzona para a riqueza do gênero em Pernambuco. Esta representatividade da subzona da mata úmida também possui correspondentes nos gêneros Heliotropium L. (Melo \& Sales 2004), Oxalis L. (Abreu et al. 2008), Phyllanthus L. (Silva

Tabela 1. Distribuição das espécies de Paspalum no Estado de Pernambuco baseada em registros de herbários, observações in loco, levantamento bibliográfico e nos registros do CNWG (Soreng et al. 2000), nas zonas e subzonas fitogeográficas de Pernambuco, segundo Andrade-Lima (1960). $\mathrm{Pr}=$ Praia; Re = Restinga; $\mathrm{Ma}=$ Vegetação Marinha; $\mathrm{Mu}=$ mata úmida; $\mathrm{Ms}=$ mata seca; $\mathrm{Mr}=\mathrm{mata}$ serrana; $\mathrm{Ag}=$ agreste; $\mathrm{St}=$ sertão; $\mathrm{Ta}=$ Tabuleiros; $\mathrm{Aa}=$ Agreste do Araripe .

Table 1. Distribution of Paspalum based on herbaria records, in loco observations, bibliographic surveys and on records of CNWG (Soreng et al. 2000), in phytogeographical zones and subzones, according Andrade-Lima (1960). Pr = Beach; Re = "Restinga"; Ma = Marine Vegetation; $\mathrm{Mu}=$ wet rainforest; $\mathrm{Ms}=$ dry forest; $\mathrm{Mr}=$ upland forest; $\mathrm{Ag}=$ "agreste"; $\mathrm{St}=$ "sertão"; Ta = "Tabuleiros"; Aa = "Agreste do Araripe".

\begin{tabular}{|c|c|c|c|c|c|c|c|c|c|c|c|c|}
\hline \multirow[b]{3}{*}{ Espécie } & \multirow{2}{*}{\multicolumn{2}{|c|}{ áxons }} & \multicolumn{10}{|c|}{ ZONA E SUBZONA FITOGEOGRÁFICA } \\
\hline & & & \multicolumn{3}{|c|}{ Litoral } & \multicolumn{3}{|c|}{ Mata } & \multicolumn{2}{|c|}{ Caatinga } & \multicolumn{2}{|c|}{ Savanas } \\
\hline & Subgênero & Grupos informais & Pr & $\mathbf{R e}$ & $\mathrm{Ma}$ & Mu & Ms & Mr & Ag & St & $\mathrm{Ta}$ & Aa \\
\hline$P$. arenarium Schrad. & Paspalum & Setacea & & $\mathrm{X}$ & & $\mathbf{X}$ & & & & $\mathbf{X}$ & & \\
\hline$P$. atratum Swallen & Paspalum & Plicatula & & $\mathbf{X}$ & & $\mathbf{X}$ & & & & & & \\
\hline$P$. calliferum S. Denham & Harpostachys & & & & & $\mathbf{X}$ & & & & & & \\
\hline P. clavuliferum $\mathrm{C}$. Wright & Paspalum & Parviflora & & & & $\mathbf{X}$ & & & $\mathbf{X}$ & $\mathrm{X}$ & & \\
\hline$P$. conjugatum $\mathrm{P}$. Berg. & Paspalum & Conjugata & & $\mathbf{X}$ & & $\mathbf{X}$ & $\mathbf{X}$ & & $\mathbf{X}$ & $\mathbf{X}$ & & $\mathbf{X}$ \\
\hline P. convexum Humb. \& Bonpl. ex Fluggé & Paspalum & Plicatula & & $\mathbf{X}$ & & $\mathbf{X}$ & $\mathrm{X}$ & & $\mathbf{X}$ & & & \\
\hline$P$. corcovadense Raddi & Paspalum & Corcovadensia & & $\mathbf{X}$ & & $\mathbf{X}$ & & & $\mathbf{X}$ & & & \\
\hline P. coryphaeum Trin. & Paspalum & Quadrifaria & & & & $\mathbf{X}$ & & & & $\mathbf{X}$ & & \\
\hline$P$. denticulatum Trin. & Paspalum & Lívida & & & & & & & & $\mathbf{X}$ & & \\
\hline P. distichum $\mathrm{L}$. & Paspalum & Disticha & & & & & & & $\mathbf{X}$ & & & \\
\hline$P$. divergens Döll & Paspalum & Caespitosa & & $\mathbf{X}$ & & & & & & & & \\
\hline P. fimbriatum Kunth & Paspalum & Fimbriata & & & & & & & $\mathbf{X}$ & $\mathbf{X}$ & & \\
\hline P. gardnerianum Nees & Paspalum & Gardneriana & & $\mathbf{X}$ & & & & & & & & \\
\hline$P$. maritimum Trin. & Paspalum & Paniculata & & $\mathbf{X}$ & & $\mathbf{X}$ & $\mathbf{X}$ & & $\mathbf{X}$ & $\mathbf{X}$ & & \\
\hline P. melanospermum Desv. ex Poir. & Paspalum & Plicatula & & $\mathrm{X}$ & & & & $\mathbf{X}$ & $\mathbf{X}$ & $\mathbf{X}$ & & \\
\hline P. millegrana Schrad. & Paspalum & Quadrifaria & & $\mathbf{X}$ & & $\mathbf{X}$ & $\mathbf{X}$ & & & & & \\
\hline P. molle Poir. & Paspalum & Caespitosa & & & & & $\mathbf{X}$ & $\mathbf{X}$ & $\mathbf{X}$ & & & \\
\hline P. multicaule Poir. & Paspalum & Parviflora & & $\mathbf{X}$ & & & & & & $\mathbf{X}$ & & \\
\hline P. nutans Lam. & Harpostachys & & & & & $\mathbf{X}$ & & & & & & \\
\hline P. oligostachyum Salzm. & Paspalum & Paniculata & & & & $\mathbf{X}$ & $\mathbf{X}$ & & $\mathbf{X}$ & & & \\
\hline P. orbiculatum Poir. & Paspalum & Orbiculata & & & & $X$ & & & & & & \\
\hline P. paniculatum $\mathrm{L}$. & Paspalum & Paniculata & & $\mathbf{X}$ & & $\mathbf{X}$ & $\mathbf{X}$ & $\mathbf{X}$ & $\mathbf{X}$ & & & \\
\hline P. parviflorum Rhode ex Fluggé & Paspalum & Parviflora & & $\mathbf{X}$ & & & $\mathbf{X}$ & & $\mathbf{X}$ & & & \\
\hline$P$. pilosum Lam. & Harpostachys & & & & & $\mathbf{X}$ & & & & & & \\
\hline P. pleostachyum Döll & Paspalum & Caespitosa & & $\mathbf{X}$ & & $\mathrm{X}$ & & & & $\mathbf{X}$ & & \\
\hline P. plicatulum Michx. & Paspalum & Plicatula & & $\mathbf{X}$ & & $\mathbf{X}$ & $\mathbf{X}$ & & & & & \\
\hline$P$. pumilum Nees & Paspalum & Notata & & $\mathbf{X}$ & & $\mathbf{X}$ & & & & & & \\
\hline P. repens P. Berg. & Paspalum & Dissecta & & & & $\mathbf{X}$ & & & & $\mathrm{X}$ & & \\
\hline P. scutatum Nees & Paspalum & Fimbriata & & & & & & & $\mathbf{X}$ & $\mathbf{X}$ & & \\
\hline P. vaginatum $\mathrm{Sw}$. & Paspalum & Disticha & $\mathbf{X}$ & $\mathbf{X}$ & & $\mathbf{X}$ & & & $\mathbf{X}$ & & & \\
\hline$P$. virgatum $\mathrm{L}$. & Paspalum & Virgata & & & & $\mathrm{X}$ & $\mathbf{X}$ & & $\mathbf{X}$ & & & \\
\hline \multicolumn{3}{|c|}{$\mathrm{N}^{\circ}$ de espécies nas Subzonas Fitogeográficas } & 1 & 17 & 0 & 21 & 11 & 3 & 15 & 12 & 0 & 1 \\
\hline \multicolumn{3}{|c|}{$\mathrm{N}^{\circ}$ de espécies nas Zonas Fitogeográficas } & & 18 & & & 23 & & \multicolumn{2}{|c|}{21} & \multicolumn{2}{|c|}{1} \\
\hline
\end{tabular}




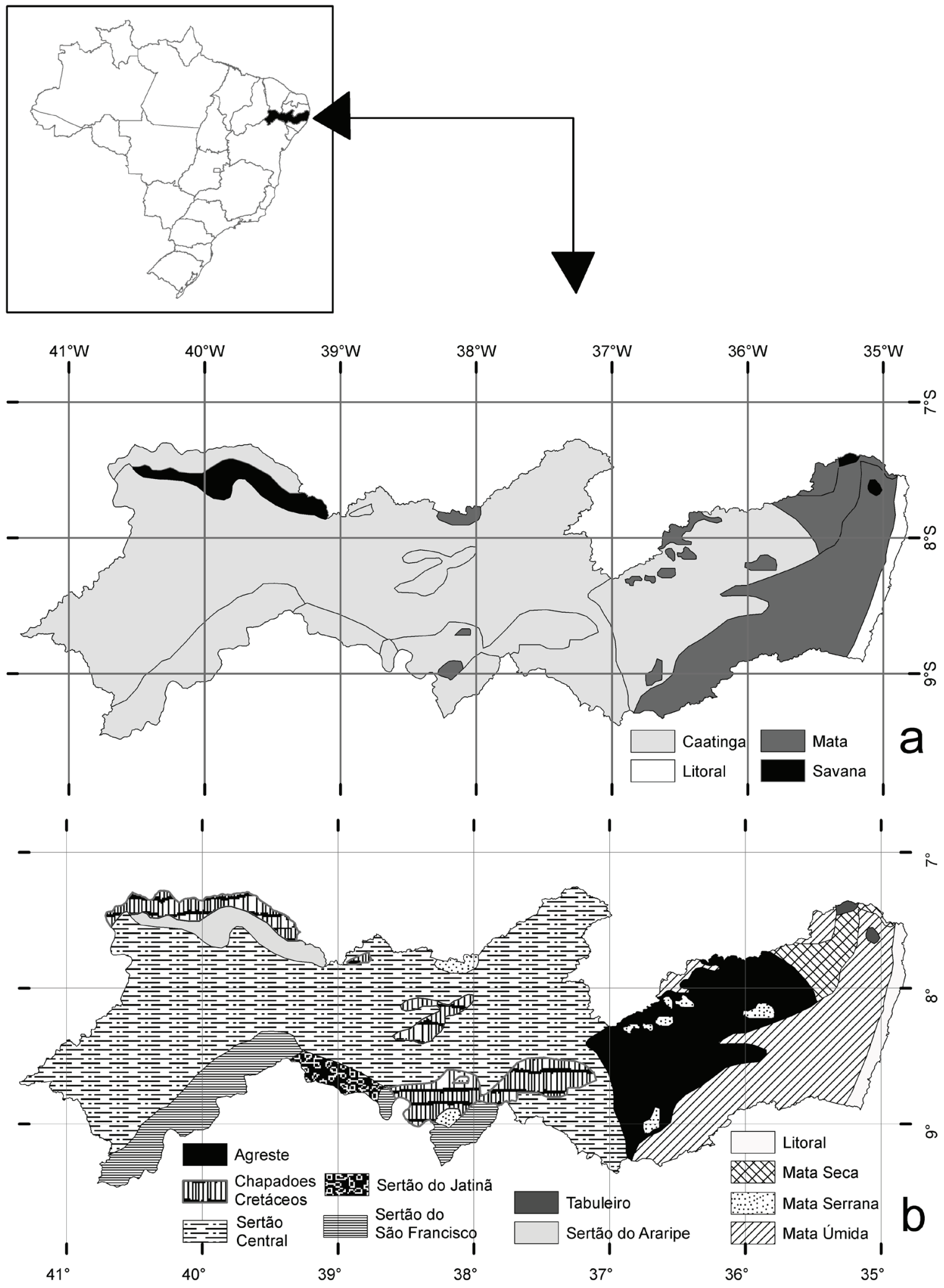

Figura 1. Localização do Estado de Pernambuco e mapas fitogeográficos mostrando as zonas e subzonas fitogeográficas de Pernambuco de acordo com Andrade-Lima (1960). a. Mapa das zonas fitogeográficas de Pernambuco. b. Mapa das subzonas fitogeográficas de Pernambuco. Legendas na figuras indicam as zonas e subzonas fitogeográficas.

Figure 1. Location of Pernambuco State and maps showing phytogeographic zones and subzones according to Andrade-Lima (1960). a. Map of phytogeographical zones of Pernambuco. b. Map of phytogeographical subzones of Pernambuco. Labels in the figures indicate the phytogeographic zones and subzones. 

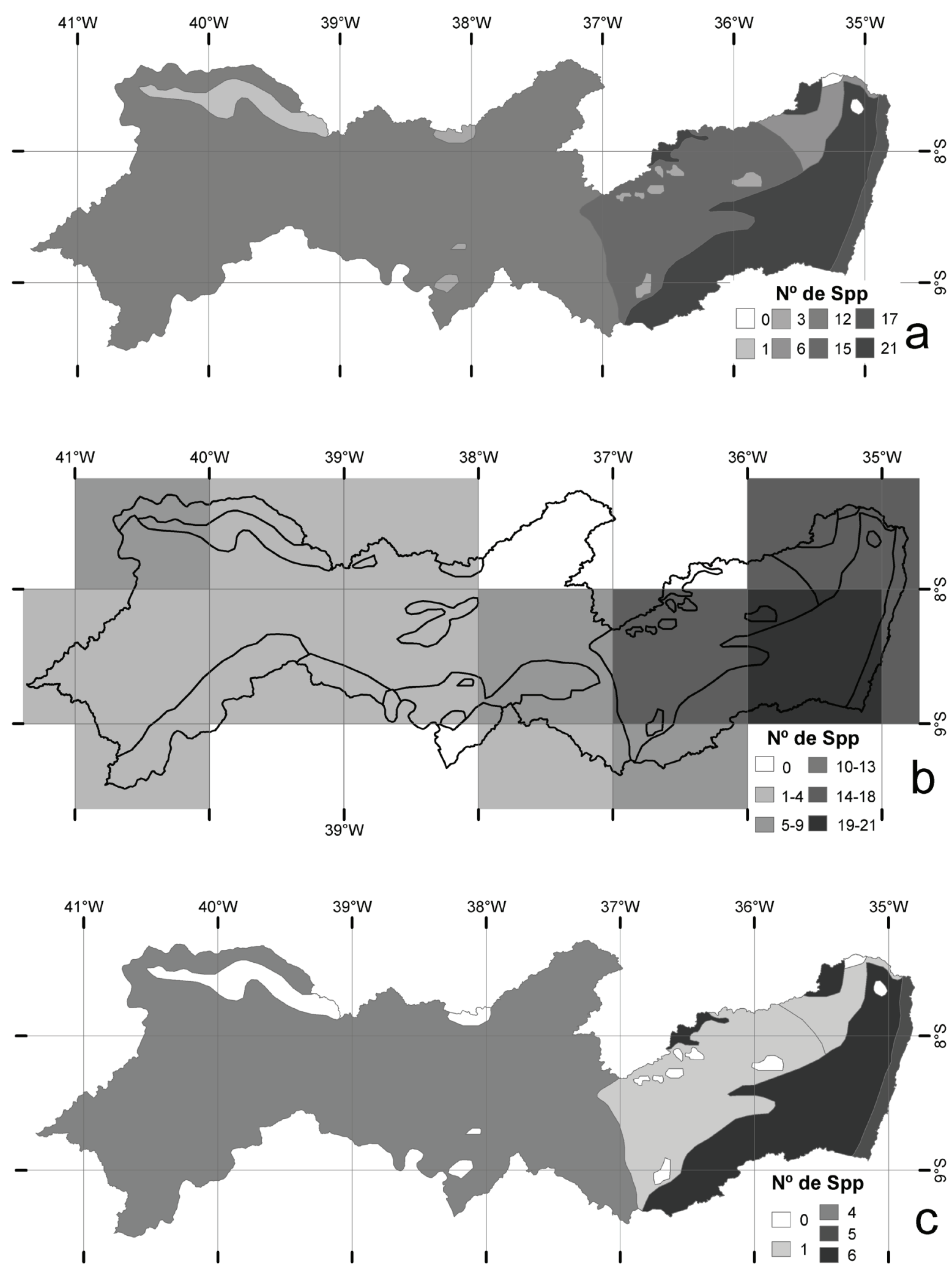

Figura 2. Mapas do Estado de Pernambuco com subzonas fitogeográficas (Andrade-Lima 1960) mostrando a distribuição da riqueza de espécies de Paspalum no Estado. a. Distribuição da riqueza de Paspalum com base nos registros de número de espécies por subzona fitogeográfica. b. Grid de $1^{\circ}$ por $1^{\circ}$ mostrando a distribuição da riqueza de espécies de Paspalum com base nos registros do número de espécies no Estado. c. Distribuição da riqueza de Paspalum por subzonas fitogeográficas com base nos registros de número de espécies classificadas como raras.

Figure 2. Maps of Pernambuco State with phytogeographical subzones (Andrade-Lima 1960) showing the distribution of species richness of Paspalum in State. a. Distribution of richness of Paspalum based on records of species number by phytogeographic subzone. b. $1^{\circ}$ by $1^{\circ}$ grid showing the richness distribution of Paspalum species based on records of species number in the State. c. Richness distribution of Paspalum by phytogeographic subzones based on records of species number classified as rares. 

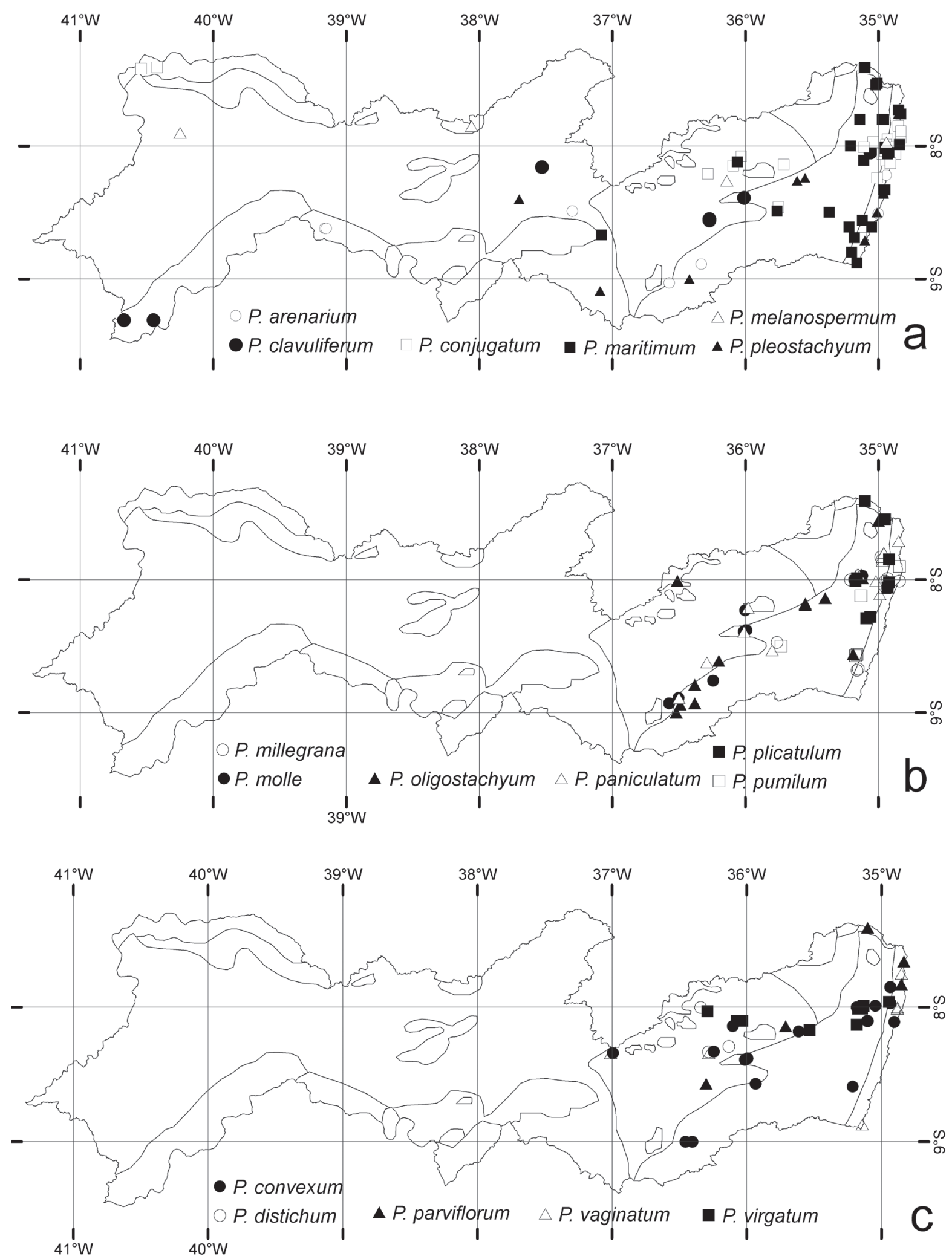

Figura 3. Mapas da distribuição das espécies de Paspalum no Estado de Pernambuco, com subzonas fitogeográficas, baseada em registros de herbários, observações in loco, levantamento bibliográfico e nos registros do CNWG (Soreng et al. 2000). a. Paspalum arenarium, P. clavuliferum, P. conjugatum, P. maritimum, P. melanospermum, P. pleostachyum. b. Paspalum millegrana, P. molle, P. oligostachyum, P. paniculatum, $P$. plicatulum, $P$. pumilum. c. Paspalum convexum, . distichum, $P$. parviflorum, $P$. vaginatum, $P$. virgatum.

Figure 3. Maps of species distribution of Paspalum in Pernambuco State, with phytogeografic subzones, based on herbaria records, in loco observations, bibliographic surveys ando n records of CNWG (Soreng et al. 2000). a. Paspalum arenarium, P. clavuliferum, $P$. conjugatum, P. maritimum, P. melanospermum, P. pleostachyum. b. Paspalum millegrana, P. molle, P. oligostachyum, P. paniculatum, $P$. plicatulum, P. pumilum. c. Paspalum convexum, P. distichum, P. parviflorum, P. vaginatum, P. virgatum. 
\& Sales 2007) e Staelia Cham. \& Schltdl. (Souza \& Sales 2004).

Seguem-se à mata úmida, em riqueza de espécies, as subzonas da restinga, com 17 espécies, do agreste, com 15 espécies, do sertão com 12 espécies e da mata seca com seis espécies. Convém ressaltar que embora a subzona do sertão seja a maior em área e extensão, ela fica apenas em quarto lugar em número de espécies. Essa distribuição segue o aumento da sazonalidade climática no sentido lesteoeste em Pernambuco que restringe a distribuição das espécies perenes, como pode ser depreendido dos dados de Andrade-Lima (1960, 1981). Tendo em vista que o ciclo de vida anual é encontrado em $27 \%$ das espécies de Paspalum em Pernambuco, é de se esperar a diminuição da riqueza na região.

Este padrão está ilustrado na figura $2 \mathrm{~b}$ que ressalta a longitude $37^{\circ} \mathrm{W}$ como um ponto de diminuição da riqueza do gênero no Estado, longitude que coincide com os limites da subzona do agreste e com o aumento da sazonalidade climática (Andrade-Lima 1960), um efeito da barreira formada pelo Planalto da Borborema para o avanço das chuvas provenientes do Atlântico, fazendo com que esta região também coincida com o limite da isoieta de $1.200 \mathrm{~mm} / \mathrm{ano}$ (Andrade 1974). Os resultados apresentados aqui reforçam a conclusão de Rodal et al. (2008) de que o Planalto da Borborema é uma barreira geográfica para as espécies da Floresta Úmida.

Muito embora seja evidente que o clima é um fator limitante para a distribuição das espécies, alguns táxons se destacam por serem encontrados ocorrendo desde as subzonas mais úmidas até as mais secas, como $P$. arenarium (figura 3a), P. clavuliferum (figura 3a), P. conjugatum (figura 3a), P. coryphaeum (figura 4b), $P$. maritimum (figura 3a), P. melanospermum (figura 3a), P. pleostachyum (figura 3a) e $P$. repens (figura $4 c$ ). A distribuição destas espécies pode ser explicada pela preferência por determinados tipos de hábitats que se repetem em distintas zonas fitogeográficas. Neste caso rios perenes, como é o caso de P. repens e solos arenosos para as demais espécies.

Esta ocorrência em hábitats semelhantes em zonas fitogeográficas distintas não invalida a proposição de que a distribuição das espécies de Paspalum em Pernambuco está condicionada ao clima, já que estas espécies com ocorrência ampla diminuem suas populações e tornam-se raras ou são encontradas apenas ocasionalmente nas subzonas mais áridas.

As espécies que ocorrem exclusivamente nas subzonas mais úmidas do Estado são $P$. atratum (figura 4b), P. calliferum (figura 4b), P. divergens (figura 4b), P. gardnerianum (figura 4c), P. millegrana (figura 3b), P. molle (figura 3b), P. nutans (figura 4b), $P$. oligostachyum (figura 3b), P. paniculatum (figura 3b), $P$. orbiculatum (figura 4c), P. pilosum (figura 4c), P. plicatulum (figura 3b) e $P$. pumilum (figura $3 b$ ). Vários autores indicaram que estas espécies têm preferência ambiental por hábitats úmidos e bordas de florestas (Barreto 1954, 1957, Canto-Dorow et al. 1996, Chase 1929, Zuloaga \& Morrone 2005), dados aqui confirmados.

Paspalum convexum (figura 3c), P. corcovadense (figura 4b), P. distichum (figura 3c), P. parviflorum (figura $3 \mathrm{c}$ ), $P$. vaginatum (figura $3 \mathrm{c}$ ) e $P$. virgatum (figura 3c) são espécies que ocorrem até a subzona do agreste, sendo os exemplos mais típicos da limitação climática da distribuição das espécies de Paspalum em Pernambuco.

Paspalum denticulatum (figura 4b), $P$. fimbriatum (figura 4a) e P. scutatum (figura 4a) ocorrem exclusivamente na zona da caatinga, sendo encontradas em todas as subzonas desta região. Merece destaque o caso de $P$. denticulatum por ser uma espécie rara no Estado e registrada para a região Sertão do Jatinã.

Com base no modelo gerado a partir da distribuição conhecida de todas as espécies, notase que existem duas regiões com elevado potencial para a ocorrência de espécies de Paspalum e que não apresentam registros (figura 5a). Uma das regiões fica ao leste, localizada num trecho que corta as subzonas da mata úmida, mata seca e agreste (figura $5 a$ ), onde foram localizadas a maioria das espécies e é uma área que concentra uma parte expressiva da população de Pernambuco e apresenta um elevado impacto na vegetação nativa promovido pela monocultura açucareira e pela urbanização (IBGE 2008). Mesmo assim, essa região apresenta importantes áreas prioritárias que servem como um direcionador de esforços para a conservação das espécies de Paspalum em Pernambuco (ver mapa do MMA 2007).

A outra região fica no lado ocidental de Pernambuco e atravessa as subzonas do sertão do Araripe e dos chapadões cretáceos (figura 5a). Vale lembrar que esta região corta o sertão do Araripe, subzona que pertence à zona das Savanas, a qual é a que mais se aproxima das outras savanas da América do Sul em aspectos fisionômicos e florísticos (Andrade-Lima 1960). É nas savanas da América do 

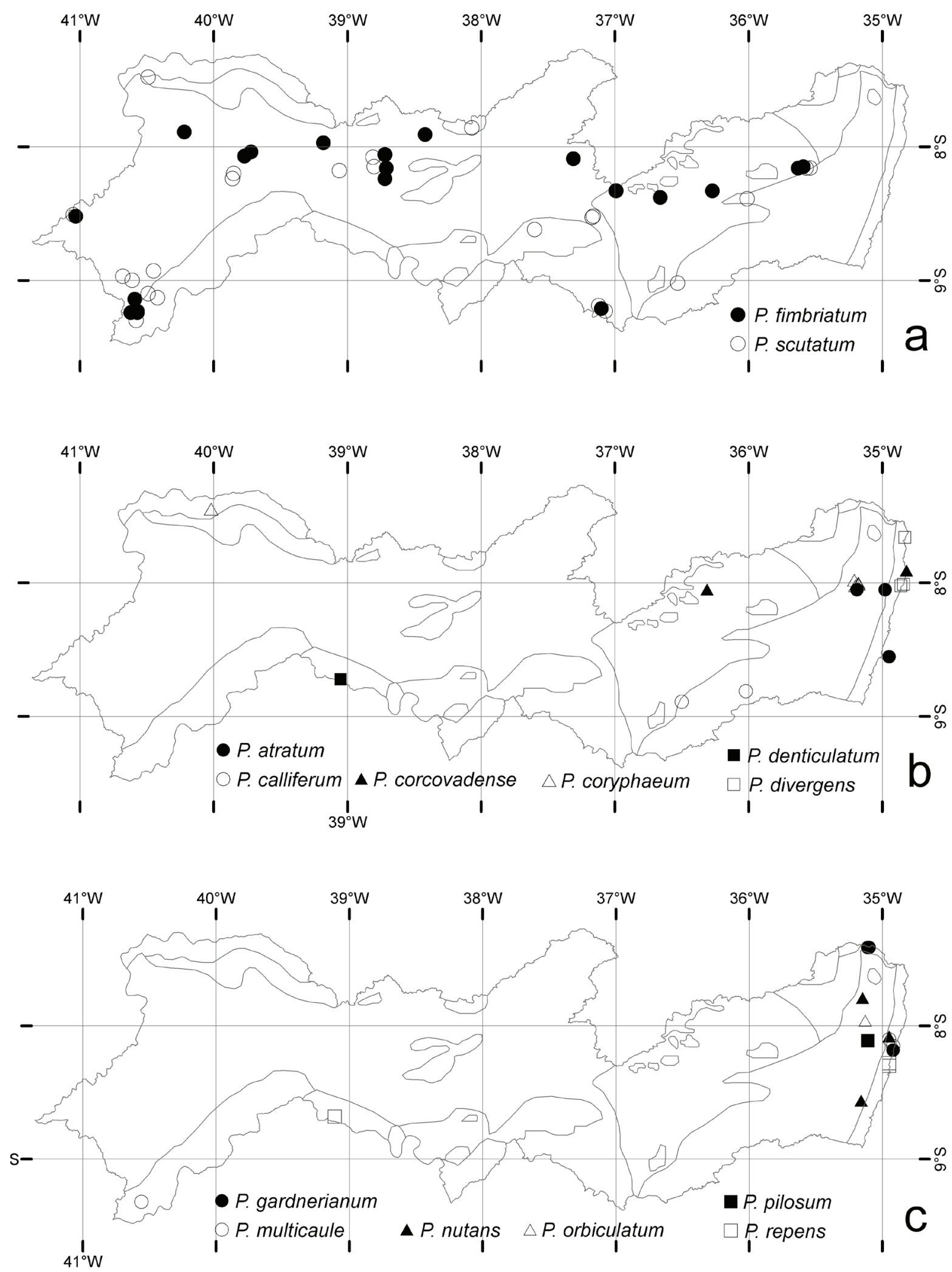

Figura 4. Mapas da distribuição das espécies de Paspalum no Estado de Pernambuco, com subzonas fitogeográficas, baseada em registros de herbários, observações in loco, levantamento bibliográfico e nos registros do CNWG (Soreng et al. 2000). a. Paspalum fimbriatum e $P$. scutatum. b. Paspalum atratum, P. calliferum, P. corcovadense, P. coryphaeum, P. denticulatum e P. divergens. c. Paspalum gardnerianum, P. multicaule, P. nutans, $P$. orbiculatum, P. pilosum e P. repens.

Figure 4. Maps of species distribution of Paspalum in Pernambuco State, with phytogeografic subzones, based on herbaria records, in loco observations, bibliographic surveys ando n records of CNWG (Soreng et al. 2000). a. Paspalum fimbriatum e P. scutatum. b. Paspalum atratum, $P$. calliferum, $P$. corcovadense, $P$. coryphaeum, $P$. denticulatum e $P$. divergens. c. Paspalum gardnerianum, $P$. multicaule, $P$. nutans, $P$. orbiculatum, $P$. pilosum e $P$. repens. 

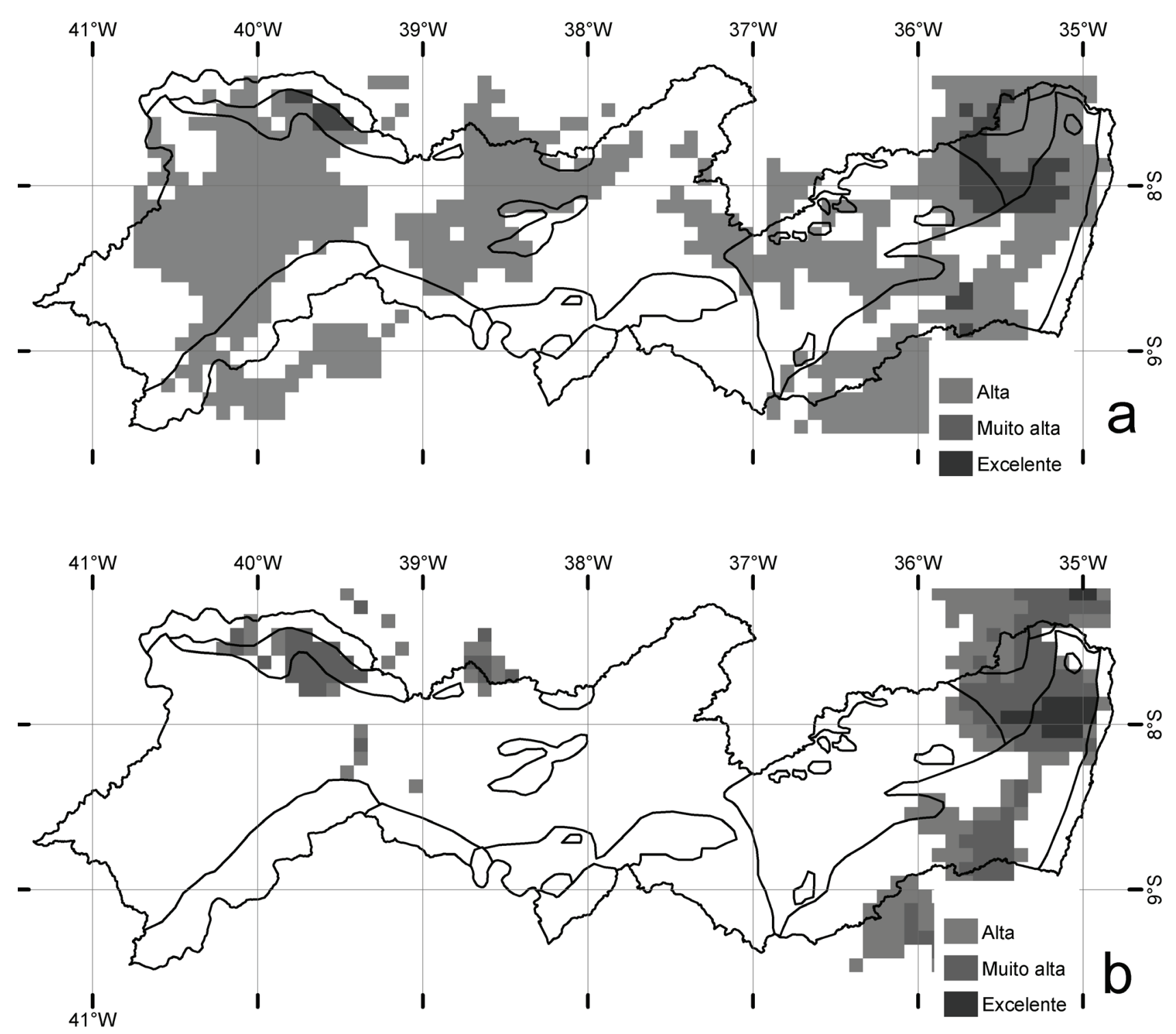

Figura 5. a. Modelagem da distribuição provável das 31 espécies de Paspalum analisadas neste estudo, com base nas 19 variáveis bioclimáticas do Worldclim. b. Modelagem da distribuição provável das espécies de Paspalum classificadas como raras ocorrentes em Pernambuco, com base nas 19 variáveis bioclimáticas do Worldclim.

Figure 5. a. Pottential distribution model of the 31 Paspalum species analyzed in this study based on 19 bioclimatics variables of Worldclim. b. Pottential distribution model of Paspalum species classified as rare occurring in Pernambuco based on 19 bioclimatics variables of Worldclim. 
Sul que o gênero Paspalum se destaca pela elevada riqueza de espécies e pelo alto nível de endemismos (Rua 2006).

Com base nos critérios estabelecidos neste estudo, foram identificadas doze espécies raras, sendo elas $P$. atratum, $P$. calliferum, $P$. corcovadense, $P$. coryphaeum, $P$. denticulatum, $P$. divergens, $P$. gardnerianum, $P$. multicaule, $P$. nutans, $P$. orbiculatum, $P$. pilosum e $P$. repens (figuras $4 \mathrm{~b}, 4 \mathrm{c}$ ). Estas espécies são encontradas nas subzonas do litoral, da mata úmida, da mata seca, do agreste e do sertão. Mas é na subzona da mata úmida onde ocorre a maioria das espécies classificadas como raras, e onde estão registradas seis espécies (figura 2c). Em termos de riqueza de espécies raras, seguem as subzonas da restinga (representado no mapa pela zona do litoral) e do agreste com cinco e quatro espécies respectivamente (figura 2c). É interessante notar que todas as espécies raras possuem suas populações registradas em áreas prioritárias para conservação das categorias muito alta e extremamente alta, conforme mapa do MMA (2007).

A concentração de espécies raras em localidades que historicamente tiveram seus territórios originais reduzidos de forma dramática e que são de elevada prioridade para conservação (Mittermeier et al. 1999, Silva \& Tabarelli 2000, Tabarelli et al. 2003) ressalta a importância destas regiões para a conservação das espécies de Paspalum registradas em Pernambuco.

No entanto é preciso esclarecer que algumas destas espécies se comportam como raras em Pernambuco, mas em outros estudos elas são tidas como espécies freqüentes ou abundantes e apresentam padrões de distribuição muito amplos (Oliveira \& Valls 2008, Morrone et al. 1996, Zuloaga \& Morrone 2005), dados que não justificariam incluílas em listas de espécies ameaçadas.

Por outro lado algumas espécies merecem atenção especial por normalmente apresentarem poucos registros e estarem em regiões susceptíveis ao impacto da ação humana (Rodriguez-Rodriguez 2003, Barreto 1965, Denham 2005). Nesse caso estão incluídas $P$. corcovadense, $P$. calliferum, $P$. divergens e $P$. orbiculatum (figuras $3 \mathrm{~b}, 3 \mathrm{c}$ ).

$\mathrm{O}$ caso de Paspalum divergens é particularmente interessante por se tratar de uma espécie que apresenta seus registros apenas para a costa leste do Brasil com distribuição conhecida até o momento para a Bahia e Pernambuco (Maciel et al. 2009b). Além de poucos registros, essa espécie possui ocorrência em locais que sofrem pressão da ocupação humana e a região dos primeiros registros dessa espécie está urbanizada e com tradicionais empreendimentos imobiliários.

Paspalum corcovadense, $P$. orbiculatum e $P$. gardnerianum são outras espécies que possuem registros antigos em áreas ocupadas pelo homem e com os hábitats já totalmente descaracterizados em Pernambuco. Essas espécies junto com $P$. divergens ocupavam o litoral das cidades de Recife, Paulista, Jaboatão dos Guararapes e Olinda. Atualmente elas estão extintas localmente, pois já não é mais encontrada vegetação nativa nessas áreas e as mesmas se mostram ineficientes como prioritárias para a conservação do gênero em Pernambuco.

A modelagem da distribuição potencial de espécies raras mostra que existe uma região considerada excelente para a manutenção dessas populações em Pernambuco (figura 5b), a qual está localizada na região metropolitana do Recife e adjacências, uma área de grande impacto antrópico, já que em seus domínios é encontrada uma população com mais de quatro milhões de habitantes (IBGE 2008). Isto reforça o quanto estas espécies apresentam suas populações ameaçadas pela perda de habitat no Estado. Pois além do desaparecimento de algumas populações registradas na década de 1930 ou 1970 (Maciel et al. 2009a), elas apresentam áreas de potencial ocorrência em locais que se encontram ocupados pela urbanização e por empreendimentos imobiliários.

O grid revela um hiato considerável no trecho que compreende a área da caatinga e volta a mostrar regiões com potencial para o estabelecimento de populações à oeste de Pernambuco, onde a subzona do sertão do Araripe se destaca como a mais promissora para a ocorrência das espécies. Convém ressaltar novamente que esta subzona é uma das que mais se aproxima em termos fisionômicos das savanas sulamericanas (Andrade-Lima 1960). Esta região também é considerada uma área prioritária para conservação proposta pelo MMA e por isto é indicada aqui como uma área para empreender um maior esforço de coleta, a fim de localizar populações para bancos de germoplasma.

A ferramenta de modelagem ambiental tem sido usada por diversos autores para predizer a ocorrência de populações que sirvam para a conservação in situ ou para a coleta de germoplasma (Jarvis et al. 2003). Neste sentido Jones et al. (1997) obtiveram sucesso em predizer a ocorrência de populações selvagens do feijão que possuíam registros na literatura mas não haviam sido coletadas. 
Os dados apresentados aqui mostram grande potencial para predizer a localização de novas populações de espécies de Paspalum para que as mesmas possam ser registradas e incluídas em coleções de germoplasma, bem como ampliado o conhecimento sobre as espécies consideradas raras.

Os estudos fitogeográficos são ferramentas importantes para analisar a distribuição das espécies e dar suporte para programas locais de conservação e manejo da flora nativa. Com os dados apresentados neste estudo, conclui-se que a distribuição das espécies de Paspalum está limitada pelo aumento da sazonalidade. Por outro lado, algumas espécies ocorrem amplamente no Estado em ambientes semelhantes em zonas fitogeográficas distintas. Em relação à conservação, nota-se que o gênero Paspalum possui espécies em Pernambuco que se encontram seriamente ameaçadas de extinção local pela destruição de seus hábitats, por este motivo as áreas prioritárias apresentadas pelo MMA para o Estado de Pernambuco se mostram eficientes para orientar a aplicação de programas de conservação deste gênero. Mesmo assim, aqui é sugerido a ampliação da área prioritária de Serra Talhada no sentido norte conectando-a com as áreas Brejo de Triunfo e Paus Brancos e ampliando no sentido oeste até atingir a porção norte de São José do Belmonte.

\section{Agradecimentos}

Os autores agradecem aos curadores dos herbários visitados em particular a Dra. Rita de Cássia Araújo Pereira (IPA) e Marlene Barbosa (UFP) que gentilmente permitiram o acesso à estrutura dos herbários IPA e UFP para a condução da maior parte deste estudo; às Dras. Silvia Terezinha Sfoggia Miotto e Reyjane Patrícia de Oliveira assim como aos dois revisores anônimos pelos valiosos comentários. O primeiro autor agradece a concessão de Bolsa de Mestrado do Programa Taxonomia CNPq/MCT. Este estudo contou com o apoio financeiro parcial da Fundação O Boticário de Proteção à Natureza.

\section{Literatura citada}

Abreu, M.C., Carvalho, R. \& Sales, M.F. 2008. Oxalis L. (Oxalidaceae) no Estado de Pernambuco, Brasil. Acta Botanica Brasilica 22: 399-416.

Andrade, M.C. 1974. Geografia de Pernambuco. Governo do Estado de Pernambuco, Recife.
Andrade-Lima, D. 1960. Estudos fitogeográficos de Pernambuco. Arquivos do IPA 5: 305-341.

Andrade-Lima, D. 1981. The Caatingas dominium. Revista Brasileira de Botânica 4: 149-153.

Arrieta, Y.H. \& Ortiz, A.C. 2009. Diversidad y distribución de las gramíneas (Poaceae) en el Estado de Zacatecas. Journal of the Botanical Research Institute of Texas 3: 775-792.

Barreto, I.L. 1954. Las especies afines a Paspalum plicatulum en Rio Grande del Sur (Brasil). Revista Argentina de Agronomia 21: 125-142.

Barreto, I.L. 1957. Las especies de Paspalum con dos racimos conjugados en Rio Grande del Sur (Brasil). Revista Argentina de Agronomia 24: 89-117.

Barreto, I.L. 1965. As espécies afins a Paspalum corcovadense Raddi (Gramineae) no Rio Grande do Sul. Revista da Faculdade de Agronomia e Veterinária 7: 197-210.

Canto-Dorow, T.S., Longi-Wagner, H.M. \& Valls, J.F.M. 1996. Revisão taxonômica das espécies de Paspalum L., grupo Notata (Poaceae Paniceae) do Rio Grande do Sul. Iheringia, série Botânica 47: 3-44.

Chase, A. 1929. The North American species of Paspalum. Contributions from the United States National Herbarium 28: 1-310.

Chiron, G.R. 2009. Riqueza e endemismo de espécies de Baptistonia (Orchidaceae), no Brasil. Hoehnea 36: 459-477.

Clayton, W.D. \& Renvoize, S.A. 1986. Genera graminum: grasses of the world. Her Magesty's Stationary Office, London.

Costa-e-Silva, M.B. \& Maciel, J.R. 2007. Os gêneros Sporobolus e Leptochloa (PoaceaeChloridoideae) em Pernambuco, Brasil. Rodriguésia 58: 147-157.

Denham, S.S. 2005. Revisión sistemática del subgénero Harpostachys de Paspalum (Poaceae: Panicoideae: Paniceae). Annals of the Missouri Botanical Garden 92: 463-532.

Hijmans, J.R.; Cameron, S.E.; Parra, J.L.; Jones, P.G. \& Jarvis, A. 2005. Very high resolution interpolated climate surfaces for global land areas. International Journal of Climatology 25: 1965-1978.

Hijmans, R.J. \& Spooner, D.M. 2001. Geographic distribution of wild potato species. American Journal of Botany 88: 2101-2112.

Hijmans, R.J., Cruz, M., Rojas, E. \& Guarino, L. 2001. DIVA-GIS, version 1.4. A geographic 
information system for the management and analysis of genetic resources data. Manual. International Potato Center and International Plant Genetic Resources Institute, Lima.

IBGE. 2008. Estimativas de população para $1^{\circ}$ de julho de 2008 enviadas para o TCU em 31/10/2008. www.ibge.gov.br/home/estatistica/populacao/ estimativa2008 (acesso em 31.03.2009).

Jarvis, A., Ferguson, M.E., Williams, D.E., Guarino, L., Jones, P.G., Stalker, H.T., Valls, J.F.M., Pittman, R.N., Simpson, C.E. \& Bramel, P. 2003. Biogeography of wild Arachis: assessing conservation status and setting future priorities. Crop Science 43: 1100-1108.

Jones, P.G., Beebe, S.E., Tohme, J. \& Galwey, N.W. 1997. The use of geographical information systems in biodiversity exploration and conservation. Biodiversity and Conservation. 6: 947-958.

Maciel, J.R., Oliveira, R.C \& Alves, M. 2009a. Paspalum L. (Poaceae: Panicoideae: Paniceae) no Estado de Pernambuco, Brasil. Acta Botanica Brasilica 23: 1145-1161.

Maciel, J.R., Oliveira, R.C \& Alves, M. 2009b. Padrões de distribuição das espécies de Paspalum L. (Poaceae: Panicoideae: Paniceae) ocorrentes em Pernambuco, Brasil. Revista Brasileira de Botânica 32: 597-605.

Melo, J.I.M. \& Sales, M.F. 2004. Heliotropium L. (Boraginaceae-Heliotropioideae) de Pernambuco, Nordeste do Brasil. Rodriguésia 55: 65-87.

Mittermeier, R., Myers, R. \& Mittermeier, C. 1999. Hotspots. Agrupación Sierra MadreCEMEX, Cidade do México.

MMA. 2007. Áreas Prioritárias para Conservação, Uso Sustentável e Repartição de Benefícios da Biodiversidade Brasileira: Atualização - Portaria MMA n $^{\circ} 9$, de 23 de janeiro de 2007. Ministério do Meio Ambiente, Brasília.

Morrone, O., Denham, S.S., Aliscioni, S.S. \& Zuloaga, F.O. 2000. Revisión de las especies de Paspalum (Panicoideae: Paniceae), subgénero Anachyris. Candollea 55: 105-135.

Morrone, O., Vega, A.S. \& Zuloaga, F.O. 1996. Revisión de las especies del género Paspalum L. (Poaceae: Panicoideae: Paniceae), grupo Dissecta (s. str.). Candollea 51: 103-138.

Morrone, O., Zuloaga, F.O. \& Carbonó, E. 1995. Revisión del grupo Racemosa del género Paspalum (Poaceae: Panicoideae: Paniceae). Annals of the Missouri Botanical Garden 82: 82-116.

Oliveira, R.C. \& Valls, J.F.M. 2008. Novos sinônimos e ocorrências em Paspalum L. (Poaceae). Hoehnea 35: 289-295.

Rodal, M.J.N., Barbosa, M.R.V. \& Thomas, W.W. 2008. Do the seasonal forests in northeastern Brazil represent a single floristic unit? Brazilian Journal of Biology 68: 467-475.

Rodriguez-Rodriguez, H.J. 2003. Sección Orbiculata (Nash) Rodriguez-R. del gênero Paspalum L. (Poaceae) en Venezuela. Ernstia 13: 105-115.

Rua, G.H. 2006. Estudos filogenéticos em Paniceae: os casos de Paspalum e Digitaria. In: J.E.A. Mariath \& R.P. Santos (orgs.). Os avanços da botânica no início do século XXI: morfologia, fisiologia, taxonomia, ecologia e genética. Sociedade Botânica do Brasil, Porto Alegre, pp. 170-173.

Silva, J. \& Tabarelli, M. 2000. Tree species impoverishment and the future of the Atlantic Forest of Northeastern Brazil. Nature 404: 72-74.

Silva, M.J. \& Sales, M.F. 2007. Phyllanthus L. (Phyllanthaceae) em Pernambuco, Brasil. Acta Botanica Brasilica 21: 79-98.

Soreng, R.J., Davidse, G., Peterson, P.M., Zuloaga, F.O., Judziewicz, E.J., Filgueiras, T.S. \& Morrone, O. 2000. Catalogue of New World Grasses. http://mobot.mobot.org/W3T/ Search/nwgc.html (acesso em 20/11/2006).

Souza, E.B. \& Sales, M.F. 2004. O gênero Staelia Cham. \& Schltdl. (Rubiaceae - Spermacoceae) no Estado de Pernambuco, Brasil. Acta Botanica Brasilica 18: 919-926.

Tabarelli, M., Siqueira Filho, J.A. \& Santos, A. 2003. A Floresta Atlântica ao norte do rio São Francisco. In: K. Porto, J. Almeida-Cortez \& M. Tabarelli (orgs.). Diversidade Biológica e Conservação da Floresta Atlântica ao Norte do Rio São Francisco. Ministério do Meio Ambiente, Brasília, pp. 25-40.

Thiers, B. 2010 (continuamente atualizado). Index Herbariorum: A global directory of public herbaria and associated staff. http://sweetgum. nybg.org/ih/ (acesso em 31.05.2010).

Zuloaga, F.O. \& Morrone, O. 2005. Revisión de las especies de Paspalum para América Del Sur Austral (Argentina, Bolívia, Sur del Brasil, Chile, Paraguay y Uruguay). Monographs in Systematic Botany from the Missouri Botanical Garden 102: 1-297. 